\title{
Ground Contact Model for Mars Science Laboratory Mission Simulations
}

\author{
Behzad Raiszadeh, David Way \\ NASA Langley Research Center, Hampton, Va., 23681
}

\begin{abstract}
The Program to Optimize Simulated Trajectories II (POST 2) has been successful in simulating the flight of launch vehicles and entry bodies on earth and other planets. POST 2 has been the primary simulation tool for the Entry Descent, and Landing (EDL) phase of numerous Mars lander missions such as Mars Pathfinder in 1997, the twin Mars Exploration Rovers (MER-A and MER-B) in 2004, Mars Phoenix lander in 2007, and it is now the main trajectory simulation tool for Mars Science Laboratory (MSL) in 2012. In all previous missions, the POST 2 simulation ended before ground impact, and a tool other than POST 2 simulated landing dynamics. It would be ideal for one tool to simulate the entire EDL sequence, thus avoiding errors that could be introduced by handing off position, velocity, or other fight parameters from one simulation to the other. The desire to have one continuous end-to-end simulation was the motivation for developing the ground interaction model in POST 2. Rover landing, including the detection of the postlanding state, is a very critical part of the MSL mission, as the EDL landing sequence continues for a few seconds after landing. The method explained in this paper illustrates how a simple ground force interaction model has been added to POST 2, which allows simulation of the entire EDL from atmospheric entry through touchdown.
\end{abstract}

\section{INTRODUCTION}

The main objective of the ground interaction model was to develop a software module within POST 2 [Ref. 1] that models the forces experienced while in contact with terrain. It was also desired that the model be simple and easy to implement. This software module has been tailored to satisfy the simulation needs of the MSL mission, but is general in nature and could be applicable to other missions. Similar to all previous Mars lander missions, MSL enters the Martian atmosphere enclosed in a capsule with the heat shield facing forward, protecting the encapsulated rover from atmospheric heating. A parachute is deployed at supersonic speeds, followed by heat shield separation. Next, the parachute and the back shell are cast away, leaving the rover with the propulsion module on top of it. The rover continues descending while rigidly attached to the propulsion module until it is close enough to ground to detach and unreel from the propulsion module. The propulsion module then "soft-lands" the rover on the ground, and flies away. The onboard controller is outfitted with the capability to detect when the rover is resting on the ground, since the propulsion module is no longer required to support the weight of the rover. Rover touchdown detection initiates the propulsion fly-away maneuver, where three bridle lines and an umbilical are severed, the propulsion module turns away from the landing site, and flies a safe distance away. The POST 2 simulation tool for MSL has all the flight software and controller code integrated within it. The ground interaction software module has been developed in order for the flight code within the simulation to detect rover touchdown. The ground interaction model described in this paper is simplistic in nature since it is not intended to 
be the prime simulation tool for evaluating the behavior the rover's mobility system during landing or determining the loads experienced during the touchdown event. Likewise, it is not meant to be the prime simulation for determining the proper settings for the part of flight code responsible for touchdown logic. Other multi-body tools simulate the rover touchdown in great detail and include appropriate soil properties and accurate models of the various joints and constraints of the MSL mobility system [Ref. 2]. The purpose of the POST 2 ground contact model is to have a very basic framework in place in order for the simulation to continue through touchdown and the propulsion module fly-away, exercising the flight software and propulsion module controller from beginning to end in one continuous simulation.

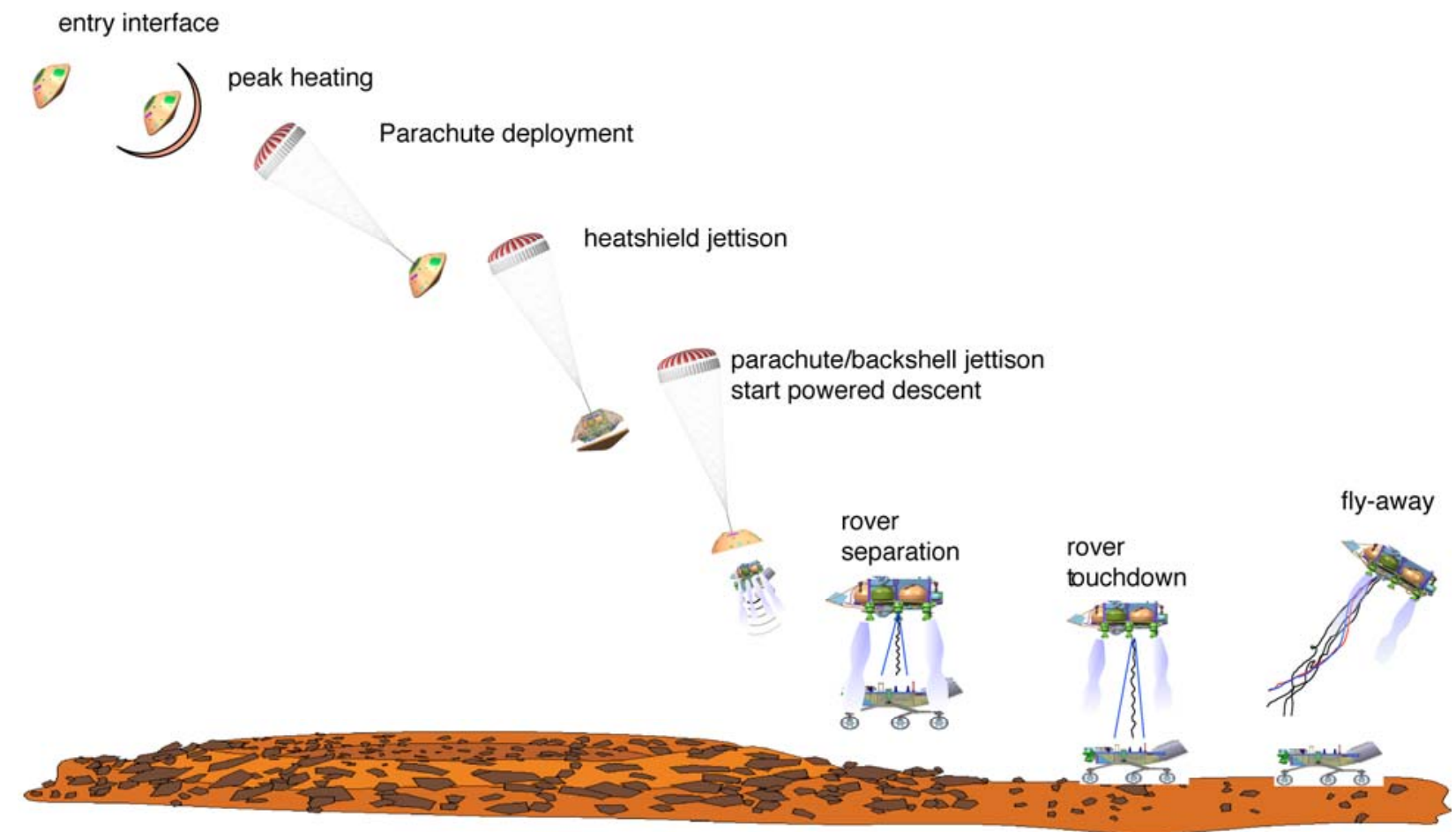

Figure 1. MSL sequence of events.

\section{METHOD}

The MSL rover is modeled in POST as a single 6 Degree Of Freedom (6 DOF) rigid body. All the flexibility within the ground interaction model is attributed to the ground, which is assumed to behave like an ideal compression-only spring-damper. The ground interaction module allows the user to designate any desired number of locations on the body to interact with the ground. For the MSL simulations, the bottom center of all six rover wheels are assumed to interact with the ground. POST 2 tracks the location and velocity of any arbitrary number of locations on the body (herein referred to as markers) accounting for the overall motion of the rover's center of mass and the additional position and velocity components due to the marker's location away from the center of mass. The ground reaction force is assumed to act perpendicular to the surface, and its magnitude is a function of how far and how fast the markers penetrate the ground model. Friction force is also being modeled to limit sliding on sloped surfaces. The type 
of friction that this paper deals with is generally called "coulomb friction," or "slip-stick friction." This friction force is always opposite to the direction the velocity vector component parallel to the plane. In other words, the friction force, opposes the in-plane component of the marker velocity vector. The resulting forces and moments from the marker are then applied to the rover's center of mass and included along with all other forces and moments acting on the rover in determining the motion of the rover. The position and velocity of the rover are then propagated as a function of time using a numerical integrator within POST 2.

\section{NORMAL FORCE}

The first part of the problem is to determine the normal force. The normal force resists the rover penetrating the surface. It is assumed that ground penetration behaves like an ideal spring damper. The normal spring force is proportional to the amount of ground penetration and the damping force is proportional to the rate of penetration. A planet fixed coordinate system is used in all the analysis to follow. In the ground coordinate system, the x-axis points north, the $y$ axis points east, the $\mathrm{z}$-axis completes the right hand system and is pointed down. The Mars terrain is assumed to be represented by a collection of flat polygons. As the rover continues descending towards the surface, the simulation software determines which polygon has been penetrated. The ground interaction model needs the planet fixed coordinates of one point on the surface and a unit vector defining the surface polygon normal direction.

The user defines the positions of the markers in the body coordinate system of the rover. POST 2 performs the proper transformations internally to find the position and velocity vector of all markers in the ground coordinate system based on the rover's position, orientation, angular orientation, and angular velocity with respect to the ground.

A range vector from an arbitrary point on the ground to the marker is calculated by subtracting their positions in the ground coordinate system.

$$
\overrightarrow{P Q}=\vec{Q}-\vec{P}
$$

The dot product of this range vector and the supplied surface unit normal vector gives the marker height relative to the pane of the ground. A positive height denotes that the marker is above the ground, while a negative height denotes that the marker has penetrated the ground.

$$
h=\overrightarrow{P Q} \hat{n}
$$

The rate of marker penetration is the component of marker's velocity vector along the ground normal vector.

$$
\hat{h}^{Y}=\vec{V}_{G} \bullet \hat{n}
$$

The normal force is then found using the ideal spring equation.

$$
\vec{F}_{N}=\left(-K h-C h \hat{h}^{\hat{n}}\right.
$$


Note that this force is in the ground coordinate system. Conversion to the body coordinate system is discussed later.

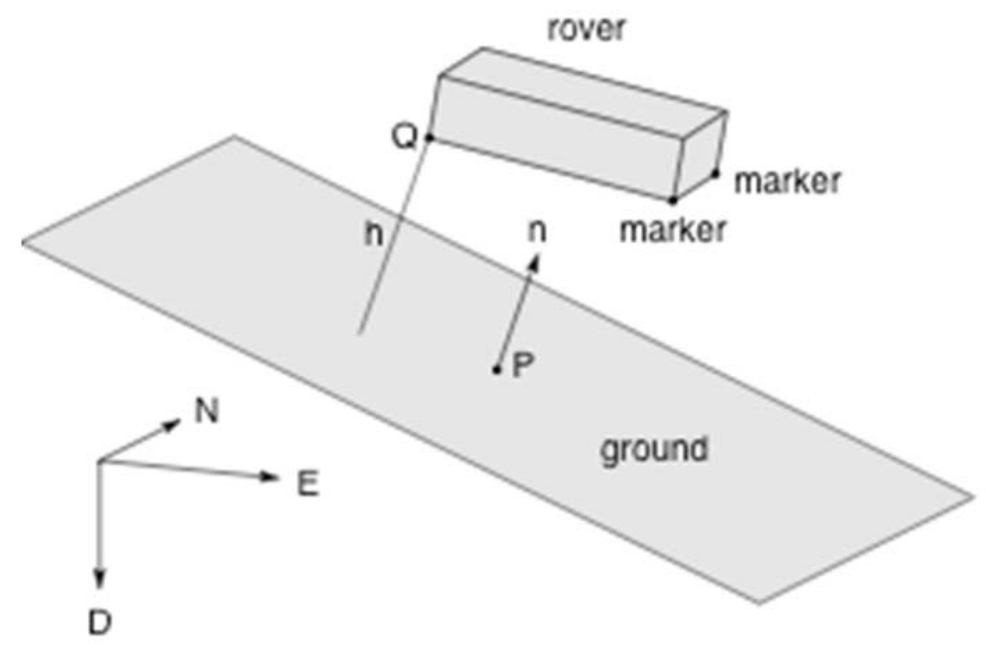

Figure 2. Ground interaction schematic.

\section{FRICTION FORCE}

The friction model assumed in this paper is called "coulomb friction", or "slip-stick friction." In such model, the friction force in a non-linear function of the relative velocity between the contacting surfaces. In order to initiate relative motion between two surfaces in contact, an outside force has to overcome the initial "stickiness." As sliding is initiated, a smaller force is required to maintain relative motion. The representation of friction as a function of relative velocity $V_{r}$ between the two contacting surfaces is shown in Figure 3 [Ref. 3].

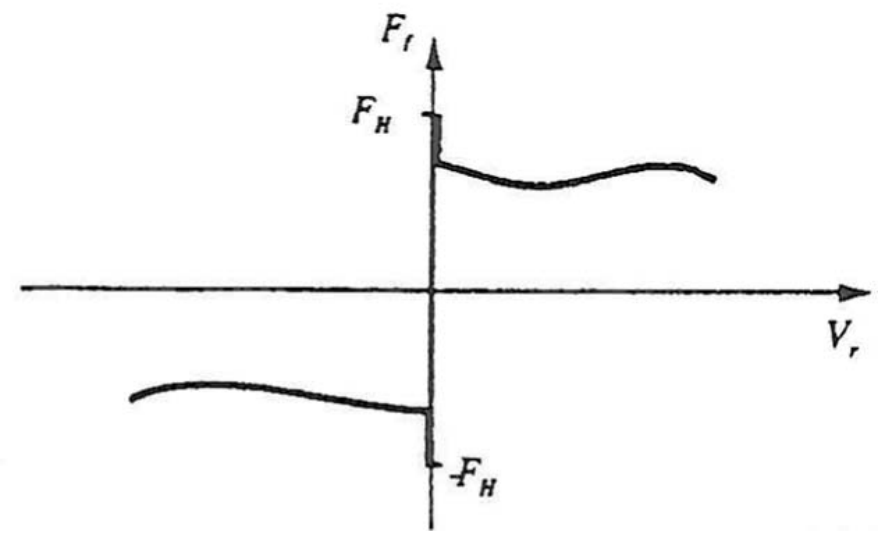

Figure 3. Standard slip-stick friction model.

Note: figure taken from Reference 3

Slip-stick friction is highly non-linear at the point $V_{r}=0$, meaning friction force $F_{f}$ could be any value in the range $\left[-F_{H},+F_{H}\right]$ that is necessary to keep surfaces from sliding. Only if the magnitude of the external forces exceeds the peak stiction force $F_{H}$, will the body begin to slide. 
The discontinuity at $V_{r}=0$ is unacceptable for purposes of simulation. Handling this discontinuity for the purposes of simulation is discussed in great detail in Reference 3 . The authors of the paper in Reference 3 ran simulations using different methods for handling friction on a mass spring-damper, and compared results. The classical method is chosen as the friction model for the MSL ground interaction simulation, mainly because of its simplicity. In the classical model, the discontinuity is approximated by a using a piecewise linear function in the region near where $V_{r}=0$ as shown on Figure 4 .

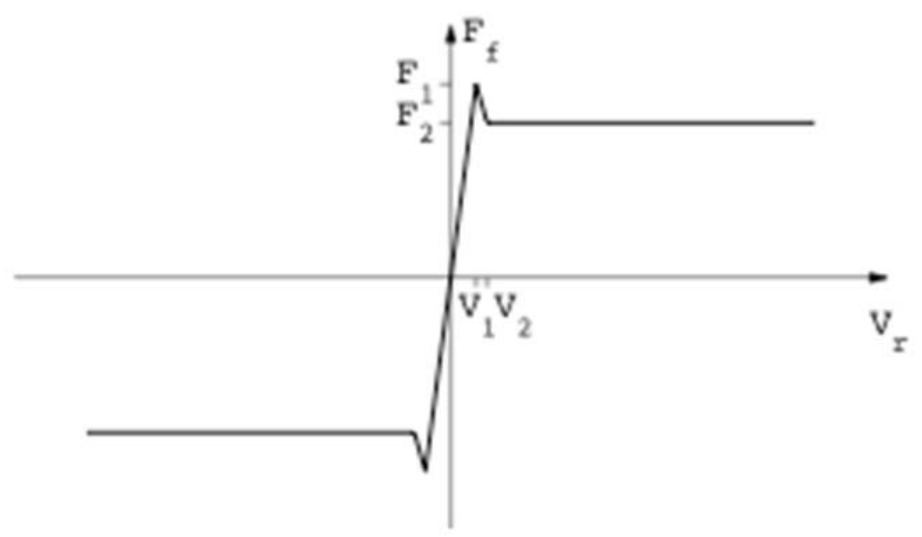

Figure 4. Classical Coulomb friction model.

Note: figure taken from Reference 3

Simulations involving the classical model are defined by two points: $\left(V_{1}, F_{1}\right)$ and $\left(V_{2}, F_{2}\right)$. There are two competing factors for the steepness of the slope defined by $\left(V_{1}, F_{1}\right)$. The slope has to be as steep as possible in order to better approximate the dynamics caused by stiction, and at the same time the slope has to be shallow enough to avoid issues caused by numerical stiffness. The best strategy is to start with a steep slope, and gradually reduce the slope until a change in the results was noticeable [Ref. 3].

The tangential velocity of the marker is needed for calculating the friction force. Tangential velocity is found by subtracting the normal component of the velocity from the marker's net velocity vector. The unit vector along the tangential velocity vector will also be needed.

$$
\vec{V}_{r}=\vec{V}-h^{Y} \bullet \hat{n}
$$

To handle the stiction condition in the simulation, the coefficient of surface friction is $\in$ assumed to behave similarly to friction force as in Figure 4. Ultimately, the friction force coefficient will have the same functional relationship to velocity as in Figure 4, because friction force and friction force coefficient have a linear relationship with each other. Therefore the user needs to supply the simulation values for $\mu_{1}$ and $\mu_{2}$, instead of $F_{1}$ and $F_{2}$. The value of $\mu_{1}$ is chosen such that the object overcomes stiction friction at a designated inclined angle, say 32 degrees, and $\mu_{2}$ is the friction coefficient such that the object maintains steady motion just overcoming the slip friction at a slightly lower surface incline angle, say 27 degrees. So, the 
simulation does a table look-up to find the friction coefficient given a tangential velocity. Friction force is then found using the classical friction equation.

$$
\begin{aligned}
& \hat{u}=\vec{V}_{r} / \vec{V}_{r} \mid \\
& \vec{F}_{F}=\mu\left|\vec{F}_{N}\right| \hat{u}
\end{aligned}
$$

The net ground force on each marker is the vector sum of normal and friction forces. Note that the net ground force vector is in the ground coordinate system. After conversion to the body coordinate system, this force, along with all other body forces, needs to be included in the numerical integration of the equations of motion. Because the point of application of the ground force is away from the center of mass, the resulting moment needs to be taken into account as well.

$$
\begin{aligned}
& \vec{F}_{G}=\vec{F}_{N}+\vec{F}_{F} \\
& \vec{F}_{B}=[G B] \vec{F}_{G}
\end{aligned}
$$

\section{GROUND INTERACTION SETTINGS}

In order to implement the ground force interaction model into the simulation, we need to choose proper settings for spring stiffness coefficient, spring damping coefficient, and the friction coefficients. In order to completely define the ground interaction model, the user has to supply four input parameters. The ground interaction input parameters are chosen so that they make physical sense and are physically intuitive to an engineer.

\section{Spring Stiffness Coefficient}

The amount of ground penetration, when the rover is fully at rest, is chosen as the parameter to describe spring stiffness. So the user supplies the ground penetration at rest, and the spring stiffness is found internally using equation 10.

$$
K=\frac{m g}{h_{e q}}
$$

\section{Spring Damping Coefficient}

The user gets to choose a damping ratio to define the damping properties of the ground interaction. Recall from basic dynamics that a simple spring-mass damper system is critically damped when the damping ratio is equal to 1.0, underdamped when damping ratio is less than 1.0, and overdamped with damping ratio of greater than 1.0. The simulation ultimately has to find the damping coefficient $C$ to use in the force calculation equations, and the user chooses the damping ratio $d$.

$$
C=2 d \sqrt{m K}
$$




\section{Friction Forces}

Two forces define the friction model described earlier, the force required to overcome the Coulomb friction $F_{1}$ and the force $F_{2}$ required to maintain steady relative motions once the Coulomb friction force has been overcome. To make this easier to implement, we ask the following two questions: 1) At what incline angle does the rover overcome the Coulomb friction, and 2) What incline angle is required for the rover to slide downhill, neither accelerating nor decelerating. Let's say that friction coefficients corresponding to $F_{1} \& F_{2}$ are $\mu_{1} \& \mu_{2}$. The incline angle at which $F_{1}$ is great enough to overcome Coulomb friction is $\theta_{1}$, and $\theta_{2}$ is the incline angle at which $F_{2}$ is equal to the downhill component of the gravity force. Thus, in the simulation, the user only has to supply these two angles, $\theta_{1}$ and $\theta_{2}$, to define the friction model.

$$
\begin{aligned}
& F_{1}=\mu_{1} F_{N} \\
& m g \sin \left(\theta_{1}\right)=\mu_{1} m g \cos \left(\theta_{1}\right) \\
& \mu_{1}=\tan \left(\theta_{1}\right) \\
& \text { similarly, } \\
& \mu_{2}=\tan \left(\theta_{2}\right)
\end{aligned}
$$

\section{Ground Interaction Settings Summary}

In summary, the user needs to supply values for four input parameters to completely define the ground interaction model, $h_{e q}, d, \theta_{1}$ and $\theta_{2}$. These four parameters were chosen because, in absence of test data, it is easy for an analyst to relate these parameters to the real world, and choose values based on engineering intuition.

\section{TEST CASES IN MATLAB}

Test cases were put together using the following ground force interaction input parameters:

$h_{e q}=0.01 \quad$ There is one centimeter of ground penetration when the rover is fully at rest.

$d=0.1 \quad$ Damping ratio of 0.1 makes the system lightly damped.

$\theta_{1}=32$ If starting from rest, the rover will stay at rest unless the incline is greater than 32 degrees, at which point it will accelerate downhill

$\theta_{2}=27 \quad$ If already in motion, the rover will neither accelerate nor decelerate when the incline angle is 27 degree. If the incline angle is greater than 27 degrees, the rover 
accelerates downhill, and will decelerate if the incline angle is less than 27 degrees.

MSL mass properties and Mars gravity are chosen for the test cases. Although the MSL rover has 6 wheels, the four outer wheels are assumed to interact with the ground in this particular test case. The rover parameters used are taken from the real MSL simulations.

Table 1. Simulation Inputs.

\begin{tabular}{|c|c|c|c|c|}
\hline Description & \multicolumn{3}{|c|}{ Value } & Unit \\
\hline rover mass & 899.18 & & & $\mathrm{~kg}$ \\
\hline mass moment of inertia about $x$ axis & 774.84 & & & $\mathrm{~kg} \cdot \mathrm{m}^{2}$ \\
\hline mass moment of inertia about $y$ axis & 571.41 & & & $\mathrm{~kg} \cdot \mathrm{m}^{2}$ \\
\hline mass moment of inertia about $z$ axis & 405.09 & & & $\mathrm{~kg} \cdot \mathrm{m}^{2}$ \\
\hline wheel $\# 1$ in body coordinate system $(X, Y, Z)$ & 0.8944 & -1.0625 & 1.1650 & $\mathrm{~m}$ \\
\hline wheel \#2 in body coordinate system (X, Y, Z) & 0.8944 & -1.0625 & -1.0950 & $\mathrm{~m}$ \\
\hline wheel \#3 in body coordinate system (X, Y, Z) & 0.8944 & 1.0625 & 1.1650 & $\mathrm{~m}$ \\
\hline wheel \#4 in body coordinate system $(X, Y, Z)$ & 0.8944 & 1.0625 & -1.0950 & $\mathrm{~m}$ \\
\hline ground to body Euler angles (yaw, pitch, roll) & $0.0-9$ & 090.0 & & deg \\
\hline acceleration of gravity & 3.7132 & & & $\mathrm{~m} / \mathrm{s}^{2}$ \\
\hline
\end{tabular}

Note that in the ground coordinate system, the x-axis points north, $y$-axis points east, and z-axis points down completing the right handed coordinate system. In the Euler angle sequence, the rotation about the z-axis is applied first (roll), followed by rotation about the y-axis (pitch), and rotation about the z-axis (yaw) completes the sequence. The rover's center of mass is the origin of the body coordinate system. Figure 5 graphically displays the set up for this test case.

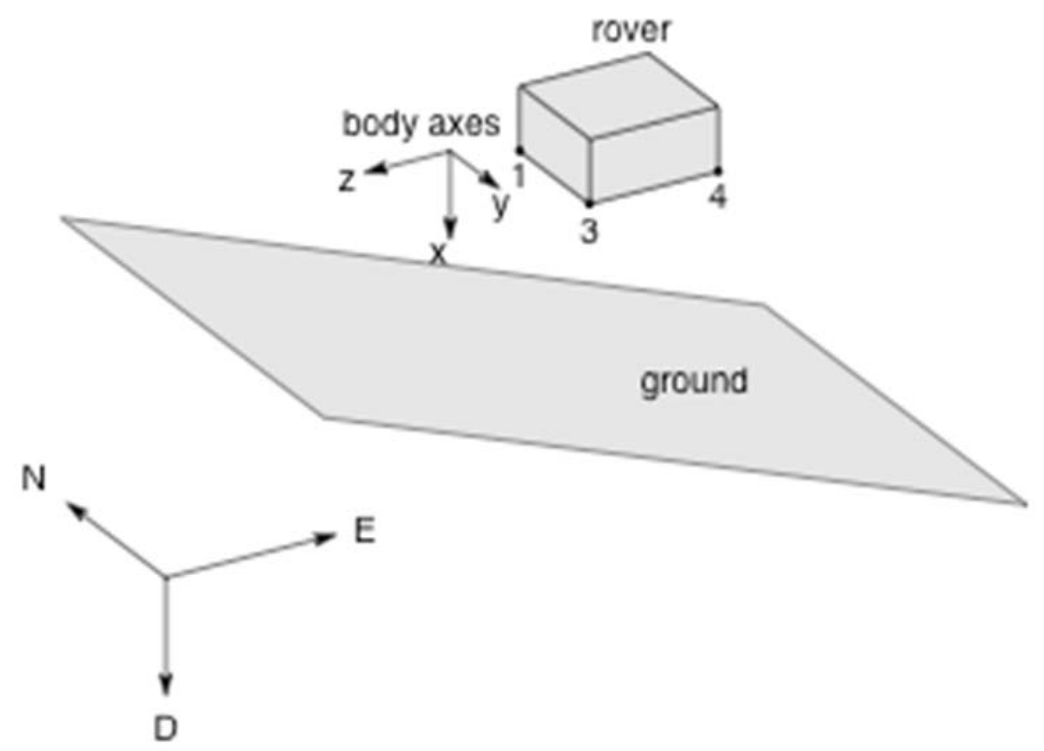

Figure 5. Test case setup. 
The simulation was run using a fourth order Runge-Kutta with an integration time step of 0.001 seconds. The rover is in an upright position with the rover orientation described by the Euler angles listed in Table 1. The rover drops straight down from rest with wheels 1 and 3 making contact with ground at the same time. Although motion in the x-axis (north) is not explicitly constrained, there is no motion in this axis. Because of symmetry, this test case can be replicated with a two-dimensional simulation. The rover's center of mass is 2 meters directly above the incline. The rover is dropped on the ground with an incline of 22 degrees. Figure 6 shows the trajectory of wheels 1 and 2 . Trajectories of wheels 3 and 4 are identical due to symmetry. Because the friction force setting $\theta_{2}$ is 27 degrees, the friction force is greater than the component of force of gravity along the incline plane. Therefore after some initial bounces, the rover starts sliding and eventually decelerates and comes to a full stop.

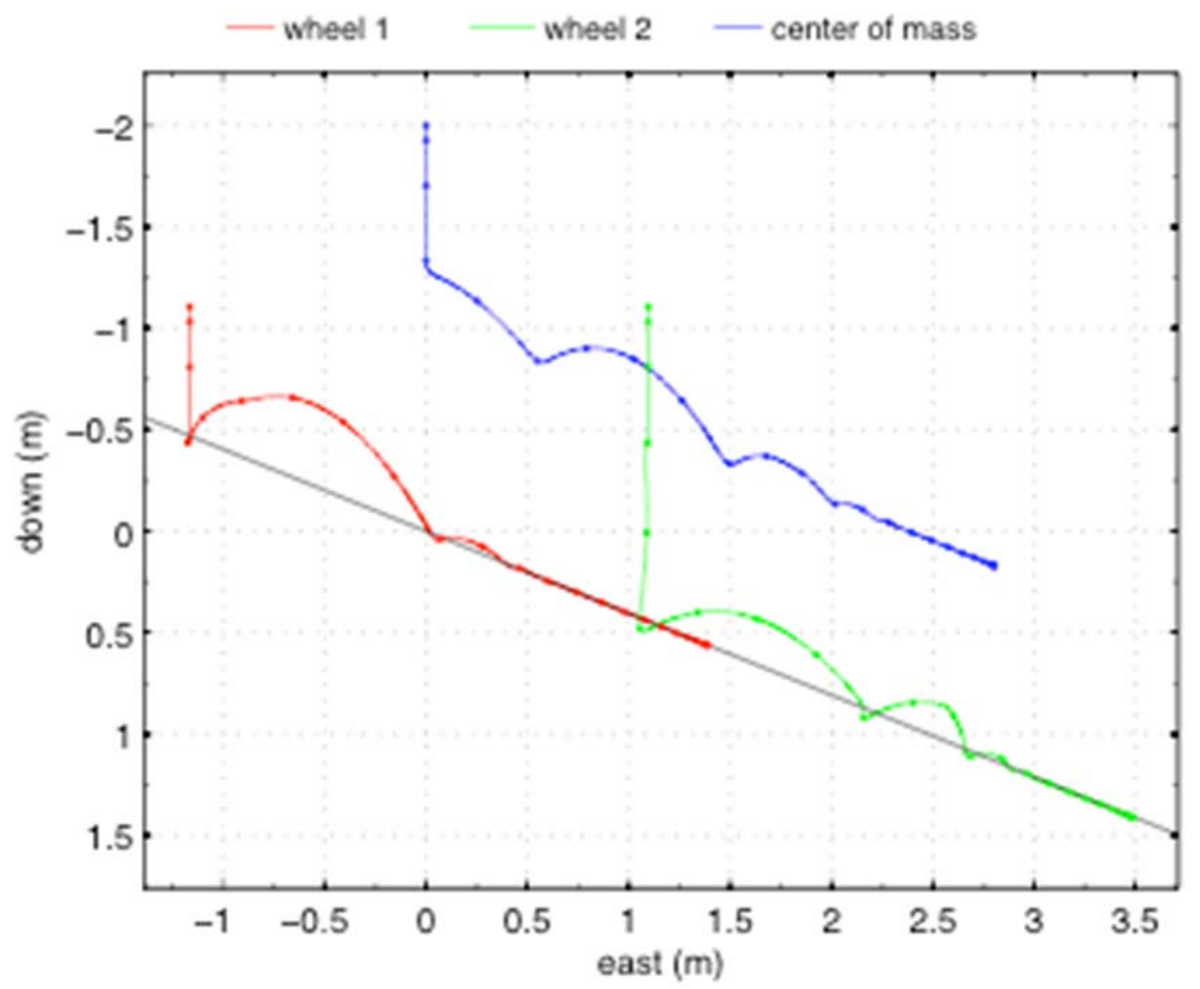

Figure 6. Trajectory of the wheels and the center of mass.

Figure 7 shows the time history of the normal force on wheels 1 and 2. Again, due to symmetry, the normal force on wheels 3 and 4 is identical to the normal force on wheels 1 and 2 . Note that the normal force on initial impact on wheel 2 is greater because wheel 2 falls further before hitting the ground. When the rover slows down and comes to near rest at the end of the simulation, it is practically in static equilibrium, therefore sum of all forces and moments need to add to zero. With all the input provided in this paper, it is possible to calculate by hand the normal force and the friction force on each wheel for static equilibrium. Solving this static equilibrium problem would make a good problem for an undergraduate statics course. The hand calculated normal forces on wheels 1 and 2 are 502.5 and $1045.4 \mathrm{~N}$ respectively. The forces at the end of simulation in Figure 7 are 502.3 and 1044.1 N. This is a very good match between the 
theory and the simulation. This means the magnitude of the normal force on the front wheels has to be almost twice that of the rear wheels for static equilibrium.

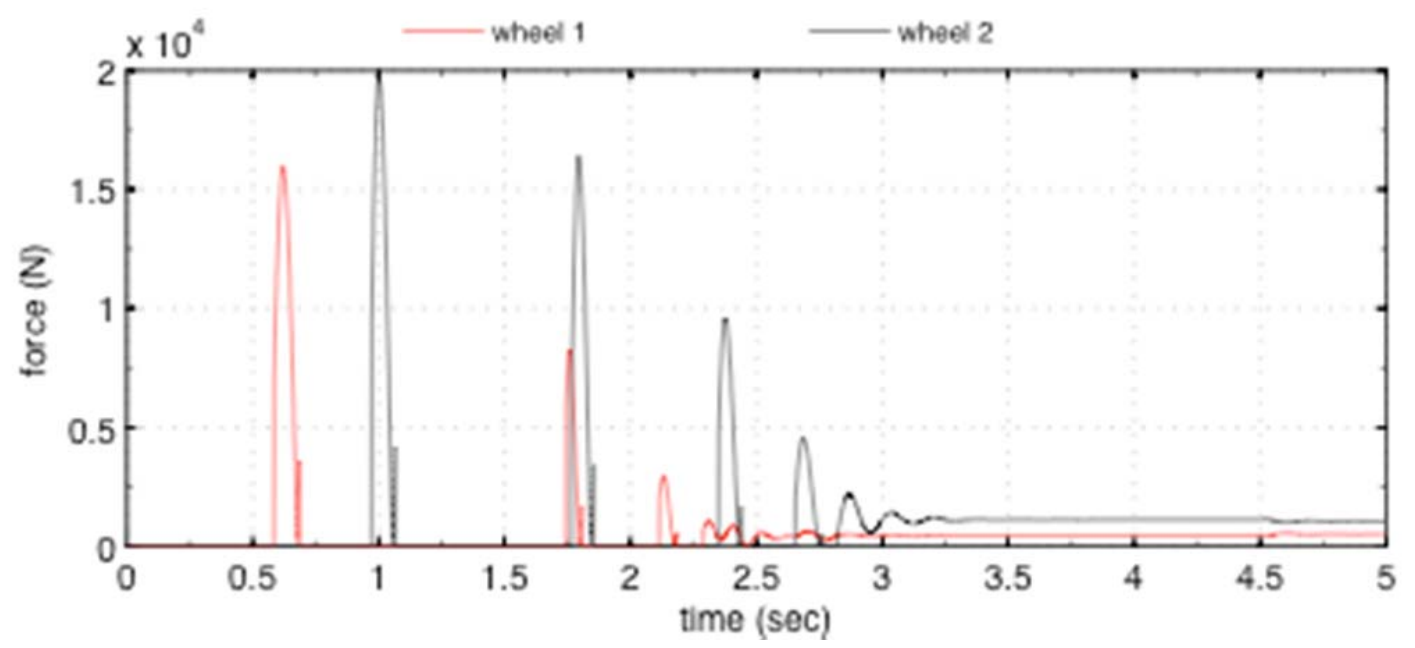

Figure 7. Force magnitude histories on wheels 1 and 2.

\section{MSL SIMULATIONS}

The purpose of this section is to demonstrate the use of the ground interaction model for MSL. The data for this section is taken from the nominal run of one of the latest Monte Carlo simulations being performed in preparation for the MSL landing on August $5^{\text {th }}, 2012$. This is a typical case and provides an example of the ground interaction model working within the POST simulation.

The touchdown trigger used for MSL is unique. This trigger is required to initiate postlanding actions, including the firing of pyrotechnic devices to sever the bridles and umbilical connecting the rover to the propulsion module and the initiation of the flyaway sequence. Previous soft-landed Mars missions (Viking and Phoenix) have utilized touchdown sensors, located on one or more legs. These sensors are designed to mechanically detect the instant of touchdown, which is a highly dynamic and transient event. The MSL touchdown trigger, however, is designed to detect not the instant of touchdown, but rather the post-touchdown state of the system [Ref. 4]. This is accomplished by monitoring the throttles of the rocket engines. When the weight of the rover is supported by the ground, rather than the rockets, a much lower throttle setting is required to maintain the proper descent rate. That is how the onboard controller knows that the rover is on the ground. 


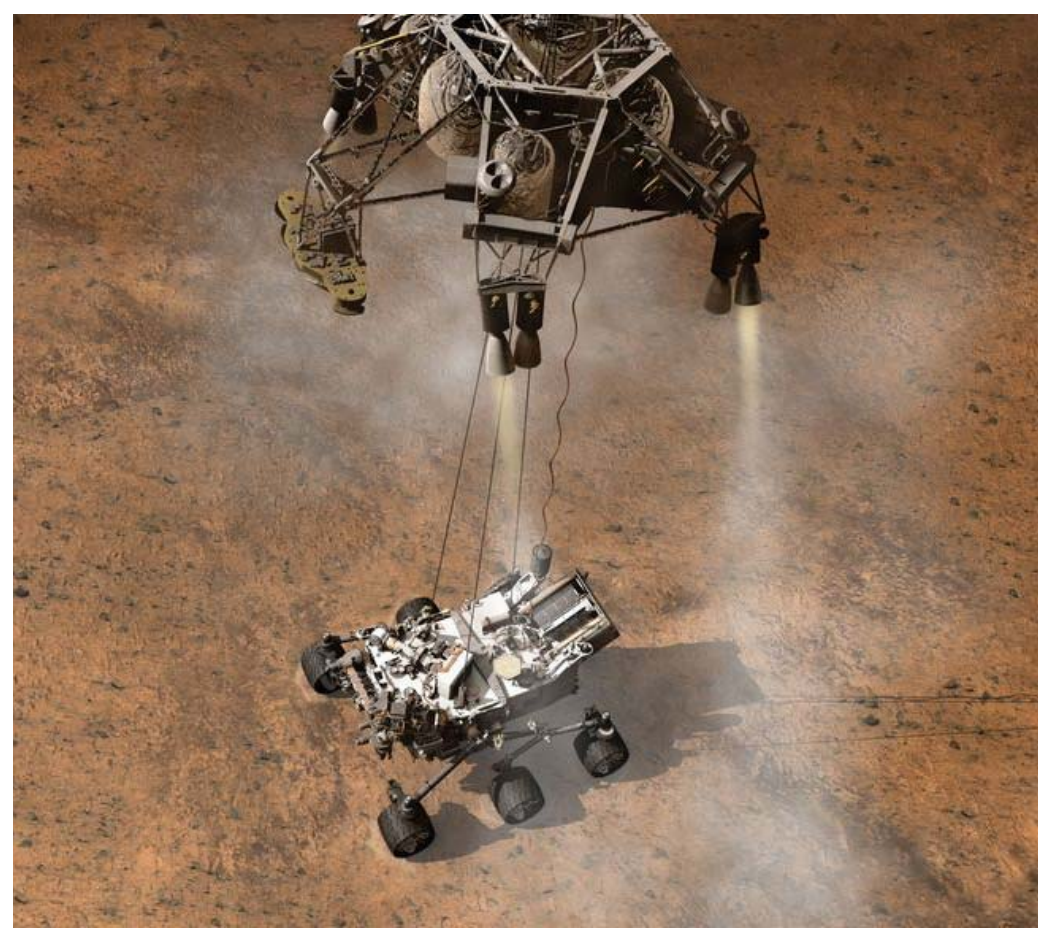

Figure 8: Artist concept of MSL landing

A Digital Elevation Map (DEM) of the Gale Crater landing site was produced at 2 x 2 meter resolution by the U. S. Geological Survey (USGS) from stereo images taken by the High Resolution Imaging Science Experiment (HiRISE) camera on board the Mars Reconnaissance Orbiter (MRO) [Ref. 5 and 6]. Figure 9 shows a portion of the DEM in western side of the landing site. This DEM is loaded into the POST 2 simulation and used to provide the individual polygons required to define the plane of the ground for the ground interaction model. On the rover, six marker positions are defined at the bottom center of each of the rover wheels. Finally, the friction coefficients were chosen to overcome stiction at slopes above 32 degrees and to continue sliding for slopes above 27 degrees. 


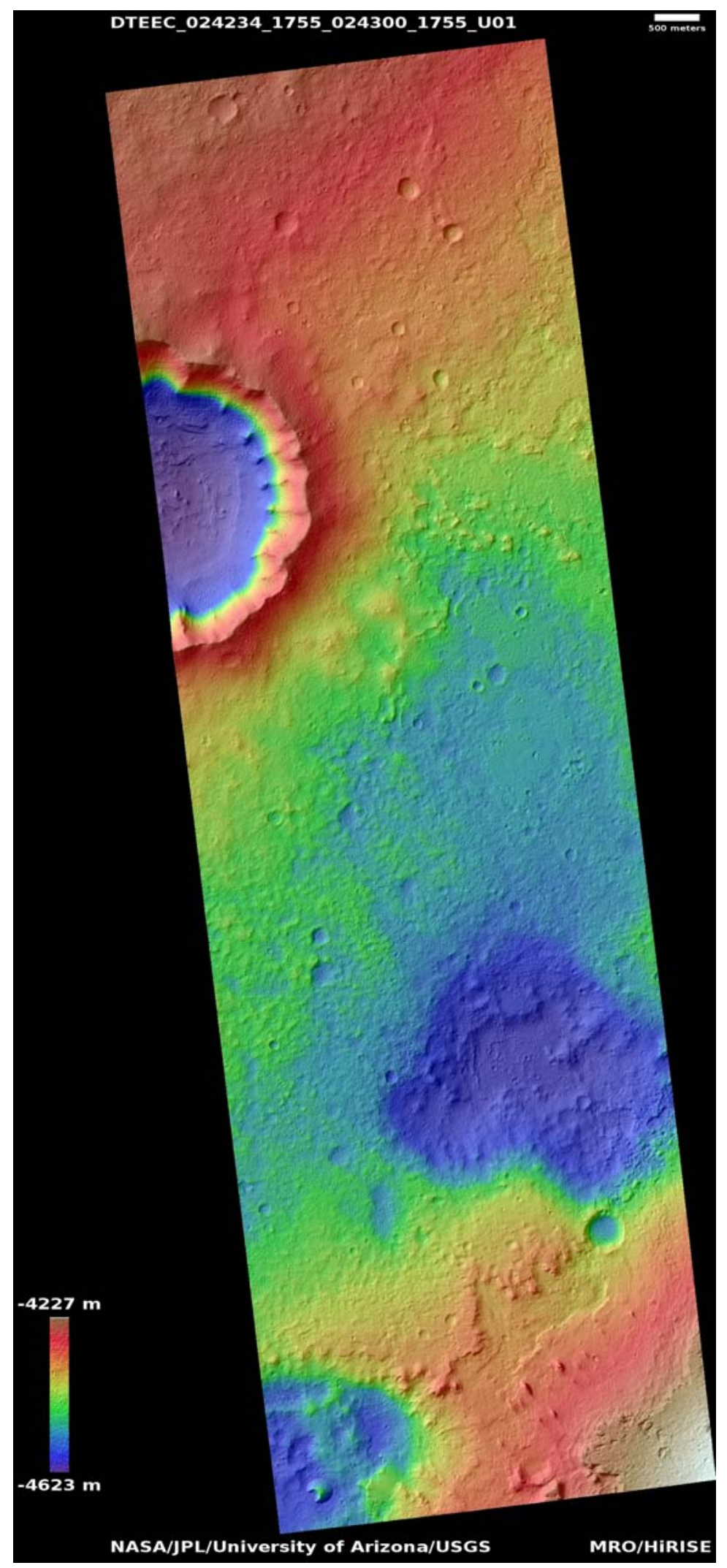

Figure 9: Gale crater digital elevation map 


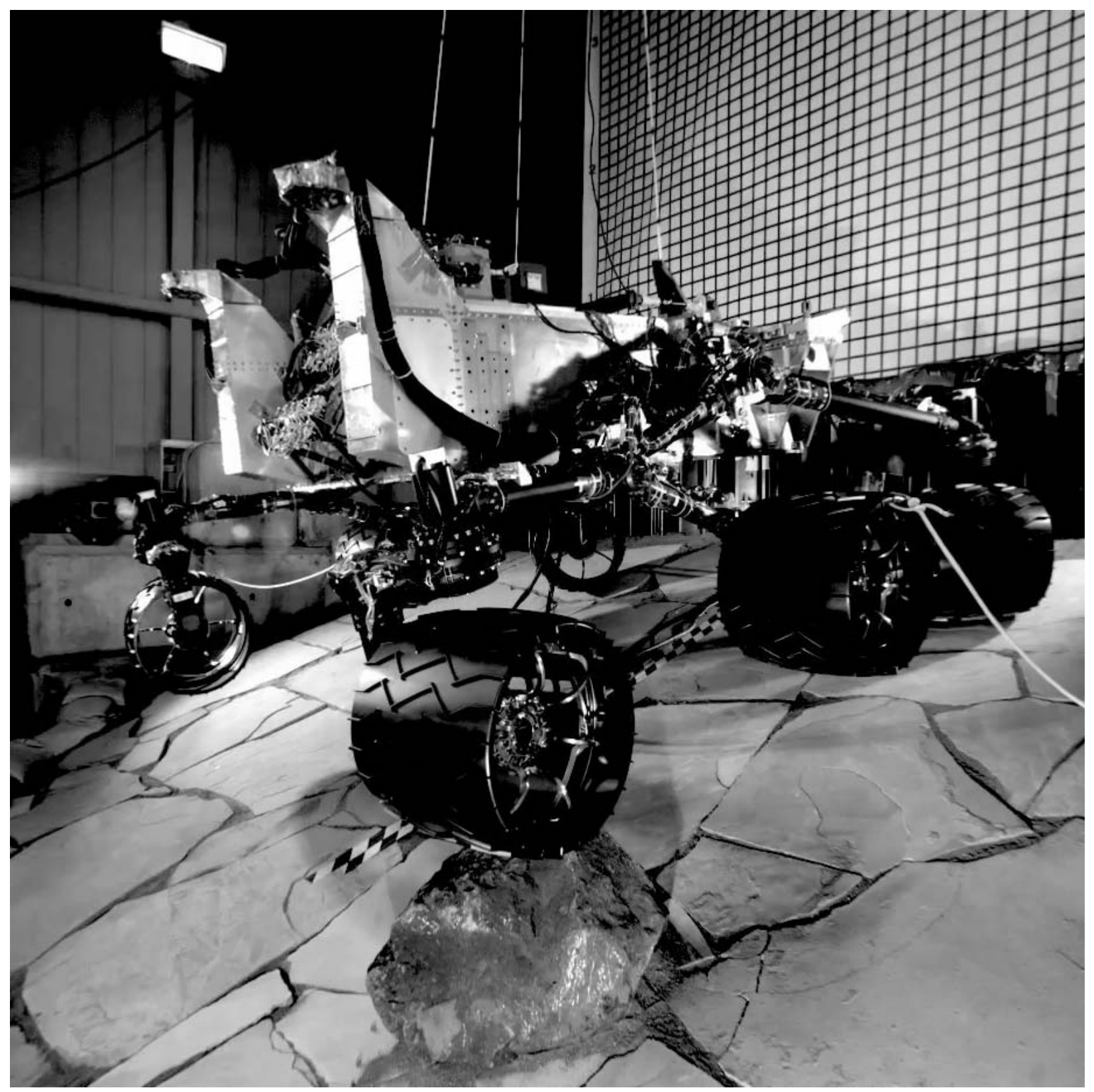

Figure 10: DTM Touchdown Testing

Figure 10 shows the MSL DTM rover during touchdown testing at the instant the aft starboard wheel strikes a rock. In this figure, the forward starboard wheel has already made contact and is resting on the surface. The three triple bridles that support the weight of the rover during Sky Crane can be seen, along with the electrical umbilical that connects the Rover Compute Element (RCE) with the Descent Stage avionics. 


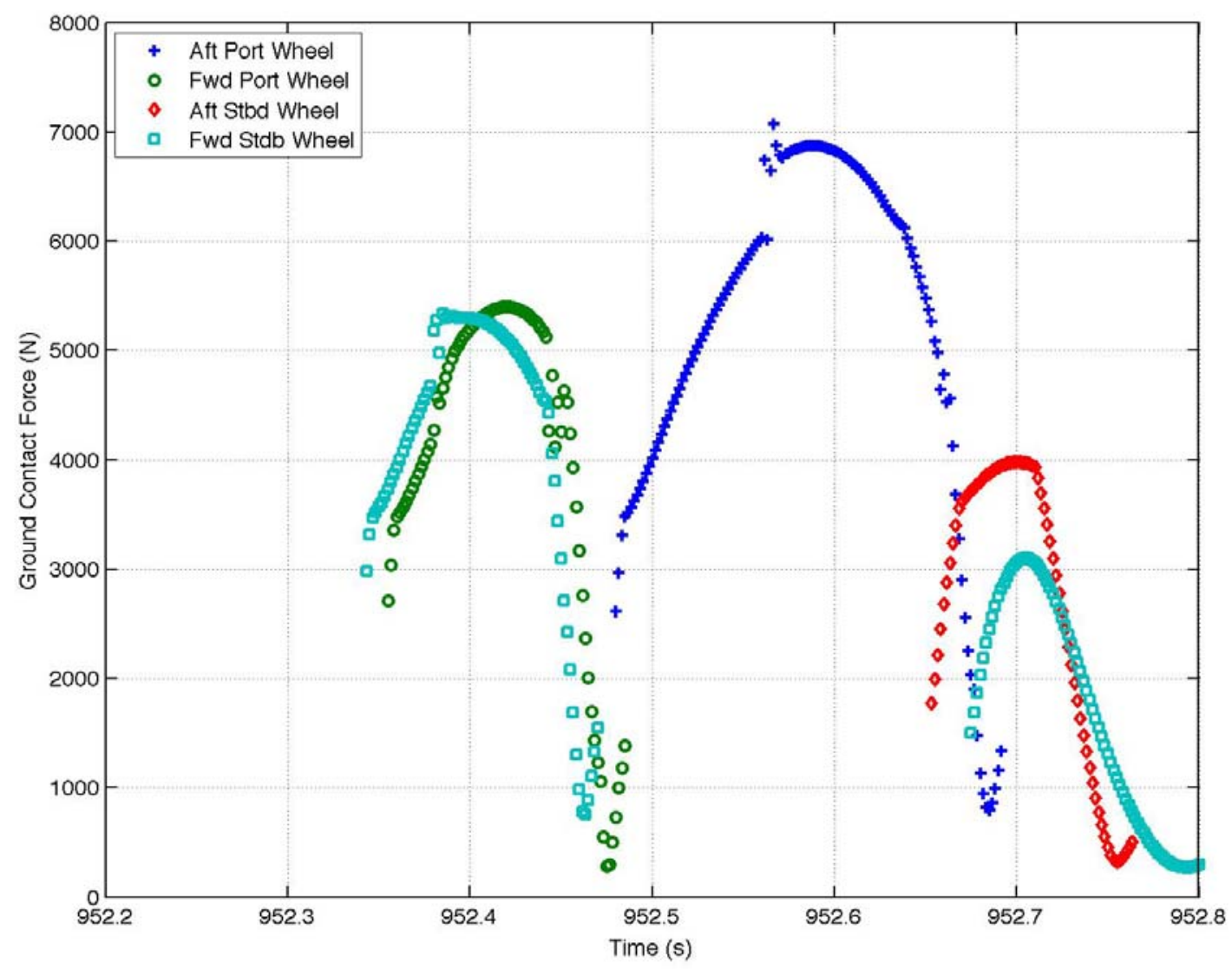

Figure 11: Ground contact forces after landing

Figure 11 shows the ground contact forces from four of the six active markers for duration of approximately half a second after initial contact with the ground. The other two middle markers, representing the wheels in the middle, did not contact the ground, due to their higher position in the touchdown pose of the mobility system. In this figure, the forward starboard wheel makes first contact at approximately 952.34 seconds. The second wheel to make contact is the forward port wheel. The rover then bounces on the forward wheels and pitches up. At approximately 952.48 seconds, the aft port wheel makes contact and the rover rolls to starboard, making contact with both starboard wheels at approximately 952.65 seconds. 


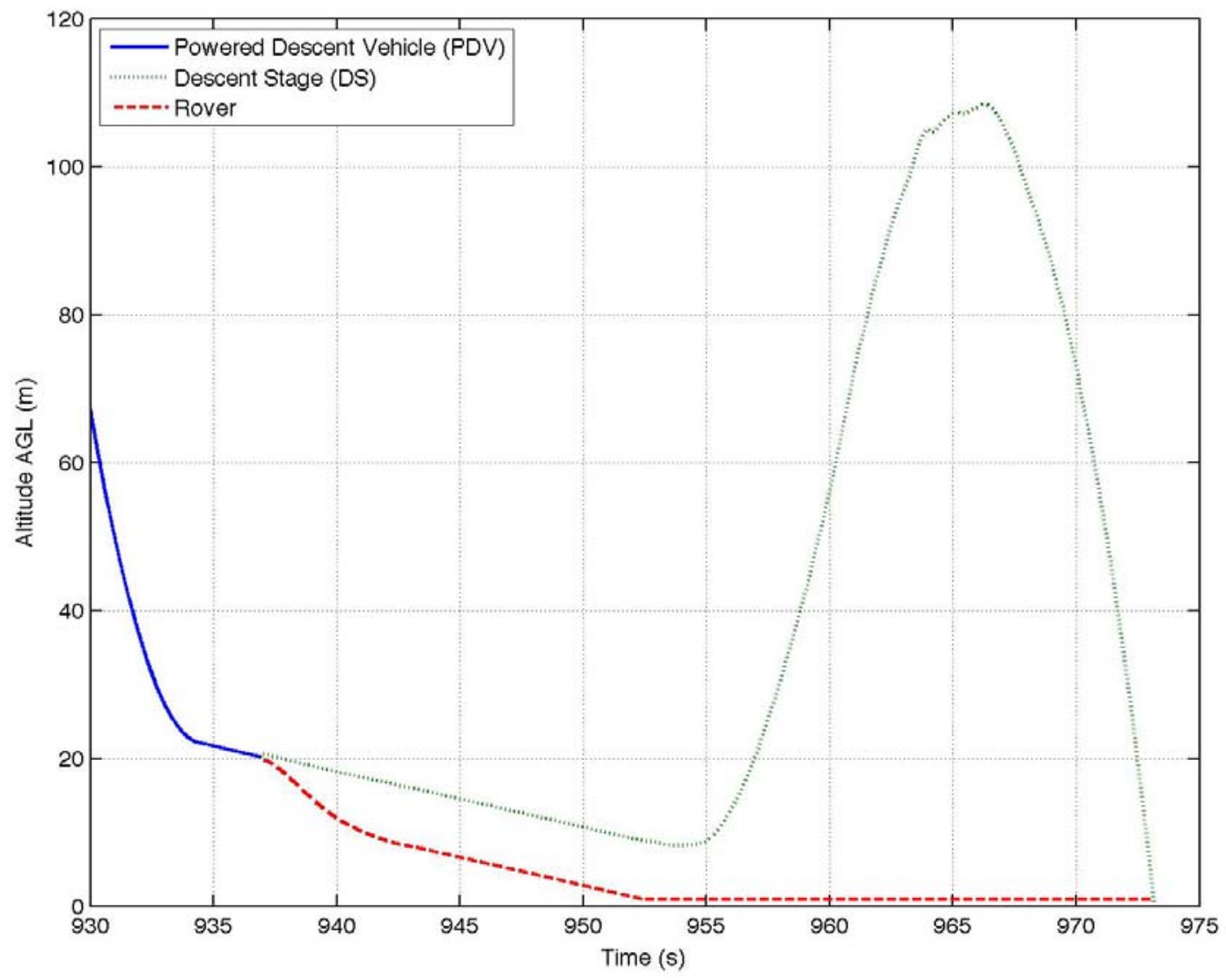

Figure 12: Altitude during MSL Sky Crane and Flyaway

Figure 12 shows the altitude vs. time trace throughout the last approximately 45 seconds of flight. This combined rover-Descent Stage configuration (called Powered Descent Vehicle, or PDV) is descending at $0.75 \mathrm{~m} / \mathrm{s}$. At approximately $20 \mathrm{~m}$ altitude, the rover is separated, and the Sky Crane mode begins. In this figure, touchdown occurs at approximately 952 seconds. Following touchdown, the Descent Stage executes the flyaway maneuver, reaching a peak altitude of just under $110 \mathrm{~m}$. During the flyaway, the rover is resting on the terrain with a constant altitude. 


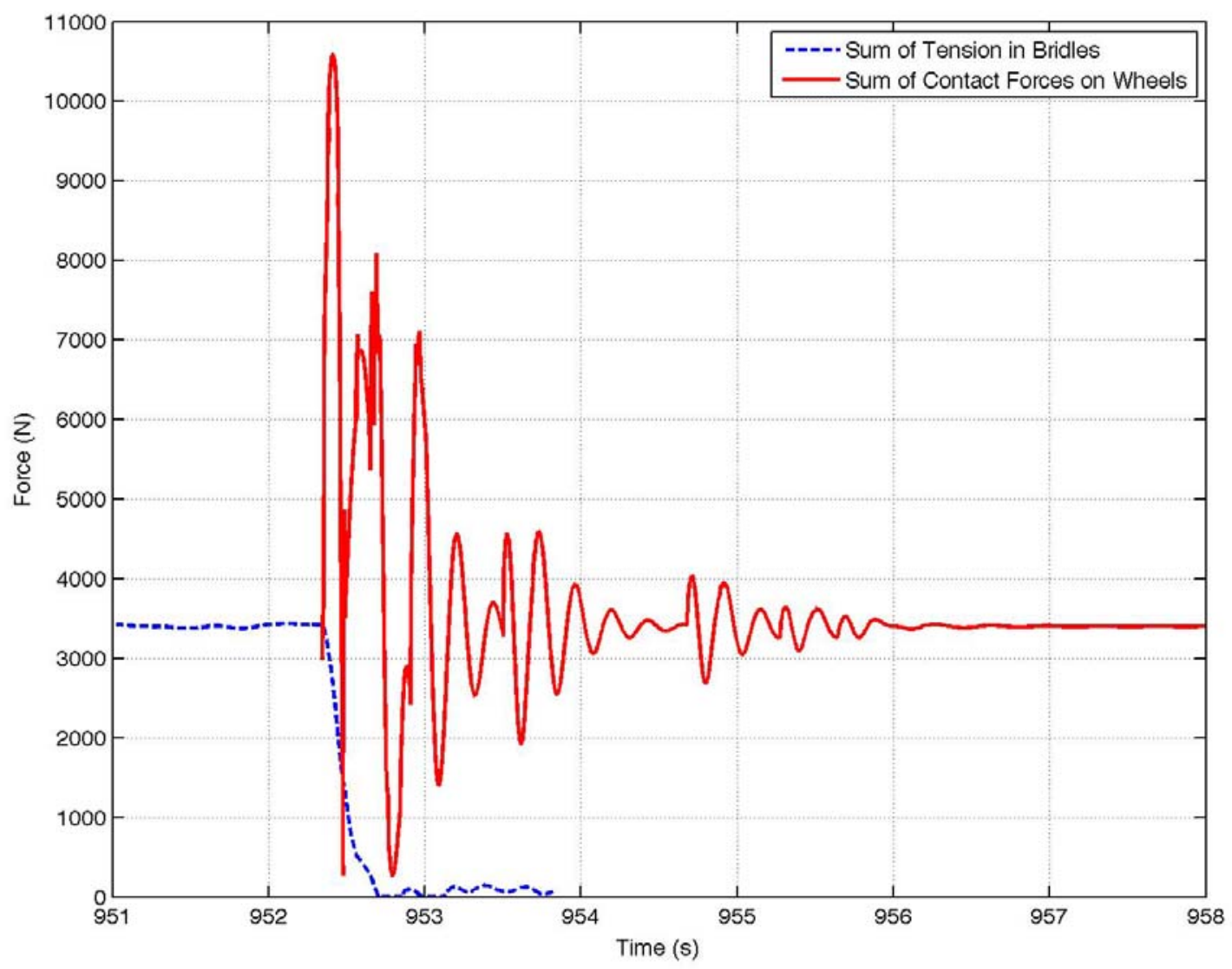

Figure 13: Bridle and Ground Forces Through Landing Event

Figure 13 shows the sum of the bridle forces and the sum of the ground forces around the touchdown event. At first the rover is not in contact with the surface and the full weight of the rover (approximately $3330 \mathrm{~N}$ ) is supported by the bridles. First contact with the surface is made at approximately 952.3 seconds. The bridle tension drops gradually as more of the rover weight is supported by the terrain. At approximately 953.8 seconds, the tension in the bridles goes to zero as the bridles are cut in preparation for flyaway. After the transient dynamics damp down, the ground forces reach a steady state $3330 \mathrm{~N}$ value as the rover comes to rest with the terrain now fully supporting its weight.

\section{CONCLUSIONS}

The methods and techniques outlined in this paper were successfully implemented in MSL end-to-end EDL simulations. This ground interaction model allowed the simulations to continue past ground impact through the flyaway maneuver and the descent stage ground impact. The EDL team ran monte-carlo simulation of the entire landing sequence evaluating all EDL flight systems including the landing system containing the ground interaction module. The ground interaction module proved to be numerically inexpensive, robust and produced results that were reasonable. 


\section{NOMENCLATURE}

C ground interaction damping coefficient

$d \quad$ ground interaction damping ratio

$F_{1} \quad$ magnitude of force needed to overcome the Coulomb friction

$F_{2} \quad$ magnitude of friction force once Coulomb friction force is overcome

$\vec{F}_{N} \quad$ normal force vector on the marker in ground coordinate system

$\vec{F}_{F} \quad$ friction force vector on the marker in ground coordinate system

$\vec{F}_{B} \quad$ net ground interaction force vector in body coordinate system

$\vec{F}_{G} \quad$ net ground interaction force vector in ground coordinate system

[GB] ground to body coordinate transformation matrix

$h \quad$ marker height from the ground

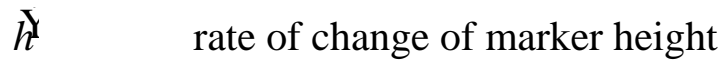

$K \quad$ ground interaction stiffness

$\vec{M}_{B} \quad$ net moment vector due to ground interaction in body coordinate system

$\hat{n} \quad$ unit vector normal to ground in ground coordinate system

$\vec{P} \quad$ coordinates of a point within the ground in ground coordinate system

$\vec{Q} \quad$ coordinates of the marker in ground coordinate system

$\overrightarrow{P Q} \quad$ vector from point on the ground to marker in ground coordinate system

$\hat{u} \quad$ unit vector in direction of the tangential velocity in ground coordinate system

$\vec{V} \quad$ marker velocity vector in ground coordinate system

$\vec{V}_{r} \quad$ marker tangential velocity vector in ground coordinate system

$\mu \quad$ coefficient of friction

$\mu_{1} \quad$ coefficient of friction at which the Coulomb friction is overcome

$\mu_{2} \quad$ coefficient of friction beyond Coulomb friction level

$\theta_{1} \quad$ the incline angle required to overcome the Coulomb friction

$\theta_{2} \quad$ the incline angle required to maintain steady motion downhill

\section{ACKNOWLEDGEMENTS}

The authors would like to thank Daniel Litton from NASA Langley Research Center who deserves credit for implementing the ground interaction model in POST 2, and verifying that it produces the correct results. 


\section{RERERENCES}

[1] R. W. Powell, S. A. Striepe, P. N. Desai, P. V. Tartabini, E. M. Queen from NASA Langley Research Center, and by: G. L. Brauer, D. E. Cornick, D. W. Olson, F. M.

Petersen, R. Stevenson, M. C. Engel, S. M. Marsh from Lockheed Martin Corporation, "Program to Optimize Simulated Trajectories: Volume II, Utilization Manual”, Version 1.1.1.G, May 2000.

[2] Chia-Yen Peng, et al, from Jet Propulsion Laboratory, “Dynamic Simulations of Mars Science Laboratory EDL Landing and Stability”, IEEE Aerospace Conference, March 710, 2007, Big Sky, MT.

[3] D. A. Haessig Jr., B. Friedland, “On the modeling and Simulation of Friction”, Journal of Dynamic Systems, Measurements, and Control, September 1991, Vol 113, pp. 355 to 362.

[4] D. Kipp, M. San-Martin, J. Esmiller, and D. Way. "Mars Science Laboratory Entry, Descent and Landing Triggers”, IEEEAC \#1445, IEEE Aerospace Conference, March 310, 2007, Big Sky, MT.

[5] R. L. Kirk, E. Howington-Kraus, D. Galuszka, B. Redding, J. Antonsen, K. Coker, E. Foster, M. Hopkins, A. Licht, A. Fennema, F. Calef III, S. Nuti, T.J. Parker, M.P. Golombek "Wall-to-wall” 1-m topographic coverage of the Mars Science Laboratory candidate landing sites, 42nd Lunar and Planetary Science Conference.

[6] R. L. Kirk, et al. (2011b) "Near-complete 1-m topographic models of the MSL candidate landing sites: Site safety and quality evaluation”, European Planetary Science Conference, v. 6. 